\title{
Evaluation System of Cross-border B2B E-commerce Website Service Quality
}

\author{
Rui DONG*, Lu-lian ZOU, Wen-zhuo ZHANG and Man-chen LI \\ No.10 Xitucheng Road, HaiDian District, Beijing, China \\ ${ }^{*}$ Corresponding author
}

Keywords: Cross-border e-commerce, B2B, E-commerce website, Service quality, Evaluation system.

\begin{abstract}
The article aims to explore the service quality evaluation system for cross-border B2B e-commerce websites. By using service quality related theories based on the classic service quality evaluation model SERVQUAL and combining the service characteristics of cross-border B2B e-commerce websites., the six dimensions and several basic indicators of the evaluation system are set and the cross-border B2B e-commerce website service quality evaluation system are established. Through the issuance of questionnaires and repeated analysis of the questionnaire data, the initial evaluation system was revised and the dimension weights were determined. A cross-border B2B e-commerce website evaluation system was established and its effectiveness and rationality were confirmed.
\end{abstract}

\section{跨境B2B电子商务网站服务质量评价体系}

\author{
董睿”, 邹璐濂, 张文卓, 李曼辰 \\ 北京邮电大学, 北京, 中国 \\ “通讯作者
}

关键词：跨境电商; B2B; 电商网站; 服务质量; 评价体系

摘要：本论文以探究跨境B2B电子商务网站服务质量评价体系为目的，借鉴了以经典服务质 量评价模型SERVQUAL为主的服务质量相关理论, 结合跨境B2B电子商务网站的服务过程及 运营特点, 重新确定了评价体系的六个维度, 重新制定了数项基本指标, 初步确立跨境B2B 电子商务网站服务质量评价体系。通过发放调查问卷以及对问卷数据的反复分析, 修正了初 期的评价体系并确定了维度权重, 建立了跨境B2B电子商务网站评价体系并证实了其有效性 及合理性。

\section{1. 引言}

随着互联网技术的日渐成熟, 人们互联网思维的不断转变, 我国的电子商务市场也得到 了极大的发展。互联网已经悄然改变了我们的生活, 不仅创造了新的商业模式, 也逐渐改变 了我们的消费习惯。回顾过去的几场电商大战, 跨境电商异军突起。跨境电商的规模逐渐形 成，流程及政策趋于完善，跨境电商企业们迅速崛起。

跨境电子商务是指分属不同关境的交易主体，通过电子商务平台达成交易、进行支付结 算, 并通过跨境物流送达商品、完成交易的一种国际商业活动。跨境电子商务冲破了国家的 界限，推动了世界经济一体化、贸易全球化进程的发展，使国际贸易走向无国界贸易，具有 十分重要的战略意义。从结构上看, 中国跨境电商的交易模式中, 跨境电商B2B交易占比达 
到 $88.5 \%$, 占据绝对优势, 是跨境电商的主力军。且从发展前景上看, $\mathrm{B} 2 \mathrm{~B}$ 是主体, $\mathrm{B} 2 \mathrm{C}$ 是补 充, B2B还有极大的发展潜力和发展需求, 重点发展B2B符合我国外贸稳增长、调结构的需要。

我国B2B跨境电商增速明显, 远大于我国的进出口贸易额增速, 这说明了B2B跨境电商已 经极大地促进了我国的外贸出口, 对我国经济推动起到重要作用。但跨境B2B和其他商业模 式仍有许多区别, 如对网站服务有更高的要求。例如对于工业品来说, 许多产品只是整个产 业链条的中间产品, 所以网站的服务质量尤为重要, 不仅包括传统意义上的在线客服和网站 运营, 还包括交易的附加服务, 即整个产品生命周期的外延服务。这决定了对跨境电商B2B 网站服务质量评价的重要性。

\section{2. 基础理论与研究方案}

\section{1 基础理论}

现今, 最被管理者和学者广泛接受和采用的一种服务质量评价体系即SERVQUAL理论, 这个理论是20世纪80年代末美国市场营销学家A.Parasuraman、Zeithaml和Berry依据全面质量 管理（Total Quality Management, TCM）理论在服务行业中提出的评价体系, 此理论将服务 质量分为五个层面: 有形性、可靠性、响应性、保障性、移情性, 每一个层面又被具体地分 为若干问题, 以调查问卷的方式, 由受访者对每个问题的期望值、实际感受值及最低可接受 值进行评分。并由其确立相关的 22 个具体因素来说明它。然后通过问卷调查、顾客打分以及 综合计算得出服务质量的分数, SERVQUAL理论的计算公式如下:

式中: $S Q=\sum_{j=1}^{5} \mathrm{wj} \sum_{i=1}^{22}(P i-E i)$

$\mathrm{SQ}$ 为感知服务质量;

$\mathrm{Pi}$ 为第 $\mathrm{i}$ 个因素在顾客感受方面的分数;

$\mathrm{Ei}$ 为第 $\mathrm{i}$ 个因素在顾客期望方面的分数 $(\mathrm{i}=1 、 2 、 3 、 \ldots . . \mathrm{n} 、 \mathrm{n}=22)$;

$\mathrm{Wj}$ 为第 $\mathrm{j}$ 个属性的权重;

再将此时的 $S Q$ 分数再除以因素数 $n(n=22)$ 、就得到单个顾客平均的Servqual分数。

最后将调查中所有顾客的Servqual分数加总再除以顾客数目 $\mathrm{m}$ 就得到该服务产品的

Servqual分数, 即: Servqual $=\left(\sum_{i=1}^{m} S Q_{i}\right) / m$

随着电子商务的兴起, 对电子商务网站进行服务质量的评价也变成了新的研究热点, 引 起了国内外许多学者的关注。在研究过程中, 学者们根据研究网站的运营特点及服务过程对 SERVQUAL量表框架中的维度分类及维度涵义进行了不同的修正, 维度中的指标内容也应根 据网站特点进行相应调整。本文将以SERVQUAL模型为基础, 借鉴前人研究并根据网络特点 以及跨境B2B商务网站与普通网站的不同之处，对量表进行修正，并确定各个维度的权重。

\section{2 研究方案}

本文将以现有理论为基础, 构建初步评价体系, 并以发放调查问卷的方式对评价体系中 涉及指标进行识别验证, 最终确立跨境B2B电子商务网站服务质量评价体系。

\section{3. 调查设计与研究方法}

\section{1 指标初步设定}

本文从SERVQUAL模型中的五个维度入手, 总结之前相关研究基础, 并与有关专家进行 探讨，初步确定了新的评价模型的六个维度以及30个指标如下（表1）: 
表1 初步跨境B2B电子商务网站服务质量评价体系

\begin{tabular}{|c|c|}
\hline 维度 & 指标 \\
\hline \multirow{5}{*}{ 网站外观 } & X1：平台产品专业性 \\
\hline & X2: 网站运行速度 \\
\hline & X3：页面美观性 \\
\hline & X4：导航易操作性 \\
\hline & X5: 网站多语种转换能力 \\
\hline \multirow{5}{*}{ 安全系数 } & X6：外贸交易支付体系 \\
\hline & X7: 海关证明资质证书等出示情况 \\
\hline & X8：保护隐私及交易安全措施 \\
\hline & X9：虚假信息举报机制 \\
\hline & X10：跨境网站连接畅通性 \\
\hline \multirow{5}{*}{ 信息质量 } & X11：产品参数完善度 \\
\hline & X12：产品信息真实性 \\
\hline & X13：信息更新及时性 \\
\hline & X14：行业资讯可靠性 \\
\hline & X15：信息翻译准确度 \\
\hline \multirow{5}{*}{ 网站管理 } & X16: 跨境争端解决机制 \\
\hline & X17：服务时间跨度性 \\
\hline & X18：客服人员专业性 \\
\hline & X19：跨境诚信体系 \\
\hline & X20: 网站世界文化关怀度 \\
\hline \multirow{5}{*}{ 业务能力 } & X21：网站专业程度 \\
\hline & X22：网站成交量 \\
\hline & X23：网站客户获得率 \\
\hline & X24：跨境供应链服务体系 \\
\hline & X25：客服人员工作能力 \\
\hline \multirow{5}{*}{ 个性服务 } & X26: 制定专属推广方式 \\
\hline & X27：推送行业资讯内容 \\
\hline & X28: 企业商业决策建议 \\
\hline & X29: 优先考虑顾客利益 \\
\hline & X30: 外贸营销咨询服务 \\
\hline
\end{tabular}

\section{2 调查问卷设计与发放}

跨境B2B电子商务网站服务质量研究需要获取网站顾客的评价数据。本论文通过发放调 查问卷的方式获取数据。2017年11月1日至2018年3月7日期间，通过线上线下两种方式发放问 卷共 250 份。调查问卷主要面向电子商务方向专家、企业采购部门工作人员、企业销售部门工 作人员、网站建设人员以及电子商务专业的学生, 占比分别约为 $5 \% 、 15 \% 、 17 \% 、 19 \% 、 44 \%$ 。 最终得到有效问卷为 208 份, 回收有效率为 $83.2 \%$ 。调查问卷主要分为两部分, 第一部分为问 卷的简要介绍以及对调查对象的基本资料的统计, 第二部分即与跨境 $\mathrm{B} 2 \mathrm{~B}$ 电子商务网站服务 质量评价相关的调查问题。问题涉及上文中提到的 6 个维度 30 个指标, 并根据李克特五点量表 法设计，问题选项用数字1到5分别表示“完全不同意”、“不同意”、“一般”、“同意”、“完全同 意”五个选项, 方便受访者为 30 个指标评分。本文将以现有理论为基础, 构建初步评价体系, 并以发放调查问卷的方式对评价体系中涉及指标进行识别验证, 最终确立跨境B2B电子商务 网站服务质量评价体系。

\section{3 研究方法}

运用SPSS 24.0软件分析数据, 统计方法主要有探索性因子分析 (效度分析) 和信度分析, 分别用于找出多元观测变量的本质结构和检验量表选项的内部一致性。 


\section{4. 实证分析与体系构建}

\section{1 探索性因子分析}

在进行因子分析之前, 首先需要对 30 个观测变量进行 $\mathrm{KMO}$ 检验和巴特利特球形检验。 $\mathrm{KMO}$ 统计量取值在 0 和 1 之间, 且 $\mathrm{KMO}$ 值越接近 1 表示变量间相关性越强, 越适合做因子分析。 $\mathrm{KMO}$ 值大于 0.9 表示非常适合，0.7以上效果一般，0.5以下不合适。本文值和巴特利特球形度 检验值如下 (表2) :

表2 KMO和巴特利特检验

\begin{tabular}{|c|c|}
\hline 合适性检验方法 & 检验值 \\
\hline KMO 取样适切性量数 & 0.884 \\
\hline 近似卡方 & 2991.189 \\
\hline 巴特利特球形度检验 & 231 \\
\hline 显著性 & 0.000 \\
\hline
\end{tabular}

由表 1 结论可知, 样本 $\mathrm{KMO}$ 值为 0.884 , 大于 0.7 且接近 0.9 , 适合做因子分析, 此外巴特利 特球形度检验值为 2991.189 , 自由度为 231 , 显著性系数为 0.000 , 表明各指标间线性关系较强, 适合做因子分析。

在SPSS 24.0中，首先运用主成分分析法对数据进行尝试性分析。运用方差最大法对初始 因子进行旋转，并删除其中公因子方差小于 0.5 或因子载荷小于 0.5 的 5 项指标，再用修正后的 指标进行探索性分析, 提取主成分, 得到方差贡献矩阵, 主成分分析提取出了主成分特征值 大于 1 的 6 个因子，且提取的因子贡献率加起来为 $67.376 \%$ ，远远超过 $40 \%$ ，第 6 个主成分之后 的成分特征值均小于 1 , 对解释原变量的贡献足够小可以被忽略，因此提取 6 个主成分合理。 数据旋转后的成分矩阵如下所示（表3）：

表3 成分矩阵

\begin{tabular}{|c|c|c|c|c|c|c|}
\hline \multicolumn{7}{|c|}{ 旋转后的成分矩阵 } \\
\hline & \multicolumn{6}{|c|}{ 成分 } \\
\hline & 1 & 2 & 3 & 4 & 5 & 6 \\
\hline 平台产品专业性 & 0.228 & 0.123 & 0.302 & 0.208 & 0.151 & 0.751 \\
\hline 网站运行速度 & 0.199 & 0.143 & 0.286 & 0.236 & 0.291 & 0.732 \\
\hline 网站多语种转换能力 & 0.201 & 0.278 & 0.236 & 0.224 & 0.136 & 0.699 \\
\hline 导航易操作性 & 0.346 & 0.047 & 0.273 & 0.122 & 0.020 & 0.895 \\
\hline 外贸交易支付体系 & 0.899 & 0.174 & 0.067 & 0.092 & 0.117 & -0.141 \\
\hline 海关证明资质证书等出示情况 & 0.874 & 0.157 & 0.287 & 0.027 & 0.155 & 0.034 \\
\hline 保护隐私及交易安全措施 & 0.899 & 0.235 & 0.151 & 0.039 & 0.115 & 0.322 \\
\hline 虚假信息举报机制 & 0.742 & 0.161 & 0.202 & 0.143 & 0.105 & 0.112 \\
\hline 跨境网站连接通畅性 & 0.799 & 0.322 & -0.053 & 0.238 & 0.306 & 0.239 \\
\hline 产品参数完整度 & 0.080 & 0.102 & 0.808 & 0.122 & 0.273 & 0.211 \\
\hline 产品信息真实性 & 0.022 & 0.200 & 0.867 & 0.294 & 0.102 & 0.191 \\
\hline 信息更新及时性 & 0.053 & -0.128 & 0.901 & 0.029 & -0.269 & -0.112 \\
\hline 行业咨询可靠性 & 0.156 & 0.239 & 0.880 & 0.299 & 0.229 & 0.301 \\
\hline 信息翻译准确度 & 0.190 & 0.378 & 0.638 & 0.089 & 0.300 & 0.289 \\
\hline 跨境争端解决机制 & 0.228 & 0.376 & 0.054 & 0.873 & 0.218 & 0.337 \\
\hline 服务时间跨度性 & 0.259 & 0.256 & 0.209 & 0.797 & 0.355 & 0.100 \\
\hline 客服人员专业性 & 0.265 & 0.130 & 0.166 & 0.733 & 0.233 & 0.199 \\
\hline 跨境诚信体系 & 0.126 & 0.146 & 0.228 & 0.714 & 0.237 & 0.301 \\
\hline 网站专业程度 & 0.163 & 0.808 & 0.126 & 0.103 & 0.029 & 0.220 \\
\hline 网站客户获得率 & 0.135 & 0.707 & 0.201 & 0.085 & 0.142 & 0.177 \\
\hline 跨境供应链服务体系 & 0.165 & 0.689 & 0.355 & 0.137 & 0.005 & 0.194 \\
\hline 制定专属推广方式 & 0.046 & 0.141 & -0.064 & 0.316 & 0.204 & 0.609 \\
\hline
\end{tabular}




\begin{tabular}{ccccccc}
\hline 推送行业资讯内容 & 0.221 & 0.311 & 0.255 & 0.192 & 0.088 & $\mathbf{0 . 7 2 4}$ \\
企业商业决策建议 & 0.189 & 0.112 & 0.310 & 0.431 & 0.015 & $\mathbf{0 . 6 9 9}$ \\
外贸营销咨询服务 & 0.070 & 0.078 & 0.411 & 0.194 & 0.291 & $\mathbf{0 . 6 3 8}$ \\
\hline
\end{tabular}

由上表可清晰区分各成分对应指标，即第一主成分在“外贸交易支付体系”、“海关证明资 质证书等出示情况”、“保护隐私及交易安全措施”、“虚假信息举报机制”、“跨境网站连接通畅 性”五个指标中具有较高载荷, 而这五个指标代表了网站为用户提供安全保障的能力, 因此命 名为安全系数。同理可知, 第二、三、四、五、六主成分分布代表业务能力、信息质量、网 站管理、个性服务和网站外观，与初步设定的维度吻合，无需更改。

\section{2 信度分析}

信度分析旨在验证选项的内部一致性以及各指标可靠性，反映了相同条件下重复测量结 果的相似度。信度测量常用Alpha系数测量, 系数值在 0 到 1 之间, 且越接近 1 可靠性越高, 值 大于 0.7 代表具有较高信度。对 6 个维度 25 项指标进行信度分析后所得数据如下表所示 (表 4 ) :

表4 信度分析

\begin{tabular}{ccc}
\hline & 可靠性统计 \\
\hline 网站外观 & 项数 & 克隆巴赫 Alpha \\
安全系数 & 4 & 0.858 \\
信息质量 & 5 & 0.947 \\
网站管理 & 5 & 0.794 \\
业务能力 & 4 & 0.881 \\
个性服务 & 3 & 0.821 \\
整体 & 4 & 0.824 \\
\hline
\end{tabular}

由表可知, 各因子克隆巴赫Alpha系数均大于 0.7 , 具有较高信度, 表明了维度与指标设 计的合理性, 无需修正。

\section{3 权重设定}

通过因子分析以及信度分析确定了六个维度的权重，即方差贡献率作为权数，经过标准 化转换之后获得权重, 具体数据如下所示（表5）:

表5 维度权重

\begin{tabular}{ccccccc}
\hline \multicolumn{7}{c}{ 主维度权重 } \\
\hline 维度 & 安全系数 & 业务能力 & 信息质量 & 网站管理 & 个性服务 & 网站外观 \\
权数 & $15.159 \%$ & $14.819 \%$ & $13.623 \%$ & $13.544 \%$ & $5.190 \%$ & $5.042 \%$ \\
权重 & 0.225 & 0.220 & 0.202 & 0.201 & 0.077 & 0.075 \\
\hline
\end{tabular}

\section{4 最终结果（图1）}

跨境B2B电子商务网站服务质量评价体系

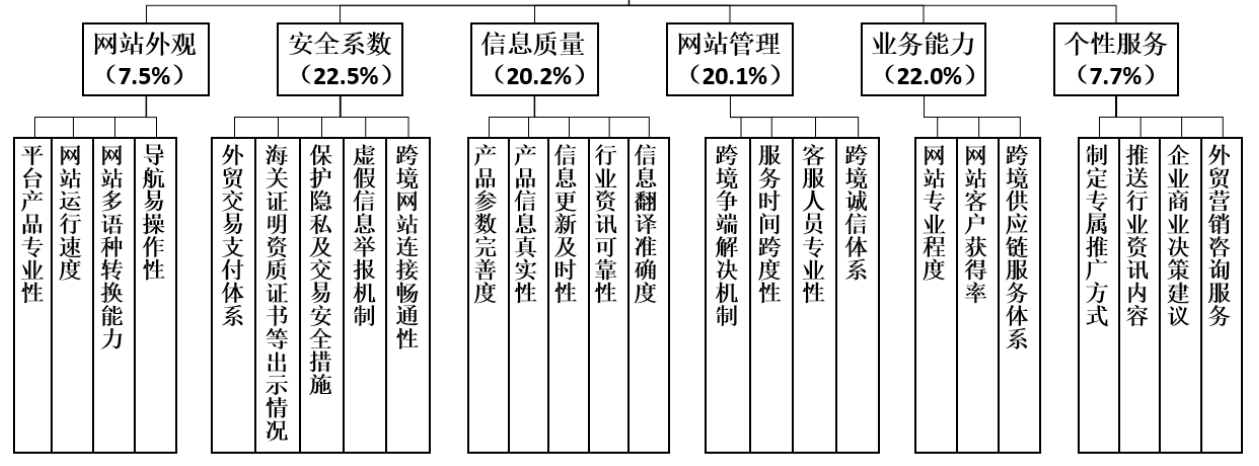

图1 最终结果图 


\section{5. 结论}

本研究构建了跨境B2B电子商务网站服务质量评价体系, 并经验证得到了6个维度25项指 标的评价工具, 在现有研究的基础上, 结合网络特点以及跨境B2B的性质与独特之处, 提出 新的思路, 增加新的内容, 为跨境B2B电子商务网站服务质量的研究提高了有意义的参考, 并能够作为实用工具供今后跨境B2B电子商务网站服务质量的评价使用。研究主要结论如下:

研究从经典服务质量评价模型SERVQUAL出发，借鉴前人研究成果，根据跨境B2B电子 商务网站的运营特点和服务方式, 设立了包括网站外观、安全系数、信息质量、网站管理、 业务能力、个性服务的六个维度, 并通过对重新定义的 25 个指标的反复验证, 证明了指标选 择的有效性和合理性。研究结果显示, 影响跨境B2B电子商务网站服务质量的六个因素的重 要性从高到低依次为安全系数、业务能力、信息质量、网站管理、个性服务及网站外观。分 析结果显示出网络保障客户安全的能力以及业务运作能力对提高网站服务质量的极为重要的 作用, 体现出了跨境B2B电子商务网站用户对网站服务质量的要求与期望, 这给跨境B2B电子 商务网站带来了更明确的目标与发展方向。跨境B2B电子商务网站应从上述六个方面出发, 正确认识自己的不足, 并采取相应措施调整改进。

\section{References}

[1] Qianqian Dong. Study on the Quality Evaluation of Tourism E-commerce Website [D]. Beijing: Beijing Institute of Technology, 2015

[2] Wenming Zuo, Wenfu Yang, Qiuping Huang, Zhenli Xie. B2C Business Website Service Quality Evaluation System and Model [J].Intelligence magazine, 2010, 29(11):82-85

[3] Tingting He, Yongjun Yan. Comparison and Analysis of Website Evaluation in China and Abroad [J].Library and Information, 2006(3):28-33

[4] Cong Zhang, Jin Yao. Evaluation System of Customer Service Quality in E-commerce Environment [J].Computer Engineering, 2005(6):66-68

[5] Fan Nana.B2B and C2C two modes of e-commerce logistics service quality evaluation index system comparison and analysis [J], Technology and method, 2015, 34(4):123-126

[6] $\mathrm{Pu}$ Yiqun.B2B website model analysis and its impact on supply chain [D]. Xiamen: Xiamen University, 2002 